\title{
Carboxyfluorescein Leakage from Poly(ethylene glycol)-Grafted Liposomes Induced by the Interaction with Serum
}

\author{
Kaname Hashizaki, ${ }^{*}, a$ Hiroyuki TAguchI,${ }^{a}$ Hideki SAKaI,${ }^{b}$ Masahiko AbE, ${ }^{b}$ Yoshihiro SAIto, ${ }^{a}$ and \\ Naotake OGAWA $^{a}$ \\ ${ }^{a}$ College of Pharmacy, Nihon University; 7-7-1 Narashinodai, Funabashi, Chiba 274-8555, Japan: and ${ }^{b}$ Faculty of \\ Science and Technology, Tokyo University of Science; 2641 Yamazaki, Noda, Chiba 278-8510, Japan. \\ Received August 8, 2005; accepted October 14, 2005; published online October 19, 2005
}

The effects of fetal bovine serum (FBS) on carboxyfluorescein (CF) leakage from poly(ethylene glycol)grafted liposomes (PEG-liposomes) were investigated. PEG-liposomes were prepared from dipalmitoylphosphatidylcholine (DPPC) and distearoyl- $N$-monomethoxy poly(ethylene glycol)-succinyl-phosphatidylethanolamines (DSPE-PEG) having PEG molecular weights of 1000, 2000, 3000 and 5000. The presence of FBS dramatically increased CF leakage from liposomes near the gel-liquid crystalline phase transition temperature, but had little effect at lower and higher temperatures. The CF leakage from PEG-liposomes whose molecular weight in PEG units was above 2000 was suppressed compared with that of liposomes without PEG. And, there was hardly any difference in the effect of the PEG molecular weight of the PEG-lipids on CF leakage from PEG-liposomes with FBS when PEG-lipids with a molecular weight in PEG units above 2000 were used. On the other hand, the leakage of CF from liposomes containing $0.145 \mathrm{~mol}$ fractions of DSPE-PEG1000 was larger than that of liposomes without PEG. Furthermore, the effects of FBS on the cooperative units of lipid molecules during the gel-liquid crystalline phase transition of liposomes were examined. However, the cooperative units of liposomes with FBS had little change compared with that of liposomes without FBS.

Key words liposome; poly(ethylene glycol); permeability; fetal bovine serum; differential scanning calorimetry (DSC) measurement; cooperative unit

Liposomes are lipids suspended in water with spherical self-enclosed systems, which have bilayer structures and an inner aqueous phase. ${ }^{1)}$ They have been used as models for biological membranes. Recently, liposomes have proven to be advantageous as carriers for drug delivery systems (DDS). ${ }^{2)}$ These advantages are biocompatibility, the ability to encapsulate both water-soluble and lipid-soluble molecules or even macromolecules, biodegradability and the ability to delivery drugs to the desired target tissues. However, liposomes are rapidly removed from the circulation following their intravenous administration, primarily by Kupffer cells in the liver and fixed macrophages in the spleen. Thus, it is important to develop modified liposomes that are able to avoid uptake by the reticuloendothelial system (RES) and extend their circulation half-life in vivo. Allen et al. ${ }^{3,4)}$ and Gabizon et al. ${ }^{5)}$ reported that the use of ganglioside $\mathrm{G}_{\mathrm{M} 1}$ as a lipid component of the liposomes allowed them to avoid or delay uptake by RES. More recently, many researchers have reported that liposomes (PEG-liposomes) conjugated with amphipathic polyethylene glycol (PEG) significantly increase the blood circulation liposome half-life compared to those without PEG. ${ }^{6-9)}$ Furthermore, the actitivity of PEG-lipid was larger than that of ganglioside $\mathrm{G}_{\mathrm{M} 1}$. The increased circulation halflife of PEG-liposomes has been attributed to the steric repulsive barrier due to the covalently attached PEG around the liposomes. ${ }^{10,11)}$

We have reported, ${ }^{12-14)}$ the effect of PEG-lipid on the physicochemical properties of liposomes. The addition of PEG-lipid to liposomes caused lateral phase separation, and the fluidity in the interfacial region of the liposomal bilayer membranes was markedly increased. In addition, the permeability of PEG-liposomes rapidly increased near the main phase transition temperature. Furthermore, the permeability of liposomes in the blood circulation is of the great interest when they are applied as drug carriers, especially for chemotherapy and gene therapy. It has been reported that many serum components interact with the liposomal bilayer membranes, in some cases causing them to release their entrapped materials more rapidly. ${ }^{15-17)}$ In this study, we investigated the effect of fetal bovine serum on the carboxyfluorescein leakage from PEG-liposomes.

\section{Experimental}

Materials $\quad$ L- $\alpha$-Dipalmitoylphosphatidylcholine (DPPC, 99.6\% pure) was obtained from NOF Co., Ltd., and distearoyl- $N$-monomethoxy poly(ethylene glycol)-succinyl-phosphatidylethanolamines (DSPE-PEG) were acquired from NOF Co., Ltd. (Tokyo, Japan). The weight-average molecular weights of poly(ethylene glycol) were 1000, 2000, 3000 and 5000. 5-(6)Carboxyfluorescein (CF, 99\% pure) was purchased from Molecular Probes, Inc. (OR, U.S.A.) and was used without further purification. Fetal bovine serum (FBS) was purchased from Sanko Junyaku Co. Ltd. (Tokyo, Japan). Dulbecco's phosphate-buffered saline (PBS) powder, composed of $\mathrm{NaCl}$, $\mathrm{KCl}, \mathrm{Na}_{2} \mathrm{HPO}_{4}$ and $\mathrm{KH}_{2} \mathrm{PO}_{4}$ was purchased from Wako Pure Chemical Industries, Ltd. (Osaka, Japan). PBS powder was dissolved in water for injection (Otsuka Pharmaceutical Co., Ltd., Tokyo, Japan), and an isotonic solution of $\mathrm{pH} 7.4$ was used. All other chemicals were commercial products of reagent grade.

Preparation of PEG-Liposomes PEG-liposomes were prepared using DPPC and DSPE-PEG, which were first dissolved in chloroform in a test tube. The solvent was then removed by blowing nitrogen gas into the test tube, and the residual solvent was further dried overnight at room temperature in a desiccator under a vacuum. PBS was added to the lipid film and warmed $\left(55-60^{\circ} \mathrm{C}\right)$ above the phase transition temperature of DPPC $\left(41^{\circ} \mathrm{C}\right)$ for $30 \mathrm{~min}$. The test tube was then vigorously shaken in a vortex mixer, and multilamellar vesicles (MLV) were obtained. Large unilamellar vesicles (LUV) were obtained from MLV suspensions extruded five times through a double-stacked polycarbonate membrane filter (pore size: $200 \mathrm{~nm}$ ) using an Extruder (Lipex Biomembranes Inc., British Columbia, Canada) at $55^{\circ} \mathrm{C}$.

Permeability Measurements To estimate the solute leakage from the liposomes, $\mathrm{CF}$ as a solute marker was encapsulated, and CF leakage was measured using a self-quenching method. ${ }^{17)}$ Briefly, the fluorescence intensity of CF is quenched at high concentration inside the liposomes, but 
emerges when the CF is released from liposomes and diluted into the external medium. Liposomes were prepared as described above, except that $50 \mathrm{~mm}$ CF was included. Liposomes encapsulating CF were separated from unencapsulated CF by gel chromatography on a Sephadex G-50 column (Amersham Pharmacia Biotech, Uppsala, Sweden) with PBS as the eluant. Leakage measurements were started by the addition of $20 \mu \mathrm{l}$ of CF-encapsulated liposomal solution to $1.98 \mathrm{ml}$ of PBS or $10 \%(\mathrm{v} / \mathrm{v}) \mathrm{FBS} / \mathrm{PBS}$ in a quartz cell (final concentration of the lipid $20 \mu \mathrm{M}$ ) at a given temperature and the fluorescence intensity of the solution was measured with a spectrofluorometer (FP-777, JASCO Co., Tokyo, Japan) equipped with a magnetic stirrer (VARIOMAG MINI, H+P Labortechnik AG, Oberschleißheim, Germany). The excitation and emission wavelengths were 490 and $520 \mathrm{~nm}$, respectively.

Differential Scanning Calorimetry The phase transition temperature of liposomal bilayer membranes was measured with a high-sensitivity differential scanning calorimeter (DSC 8230, Rigaku Co., Tokyo, Japan) equipped with a thermal controller (F-25, Julabo Labortechnik GmbH, Seelbach, Germany). The liposome solution $(20 \mu \mathrm{l})$ was put in a sampling vessel, made of stainless steel (resistance pressure: $50 \mathrm{~atm}$, vessel size: $3 \times 5(\phi) \mathrm{mm}$ ), and then the vessel was sealed. The reference was PBS with the same volume as that of the sample. When the cycle heating was carried out, samples were heated to $50^{\circ} \mathrm{C}$ and cooled to $20^{\circ} \mathrm{C}$, and then heated again. The measurement conditions were $1^{\circ} \mathrm{C} \mathrm{min}{ }^{-1}$ for the scanning rate and $35-50{ }^{\circ} \mathrm{C}$ scanning range.

\section{Results and Discussion}

Carboxyfluorescein Leakage from Liposomes When the liposome is developed as a drug carrier for DDS, it is necessary to evaluate the stability in the blood circulation. Figure 1 shows the time-courses of CF leakage from liposomes in the absence and presence of FBS. The leakage of CF from liposomes with FBS was much larger than that of liposomes without FBS at near the gel-liquid crystalline phase transition temperature of liposomal bilayer membranes (41, $\left.44^{\circ} \mathrm{C}\right)$. On the other hand, at temperatures $\left(30,50^{\circ} \mathrm{C}\right)$ away from the gel-liquid crystalline phase transition temperature, FBS hardly influences the CF leakage from liposomes.

In addition, the mechanism of CF permeation through liposomal bilayer membranes was evaluated in further detail for the rate constant of CF leakage from liposomes. It is well known that the solute leakage from liposomes follows the first order kinetics. The fluorescence intensity FI of CF at the incubation time $t$ can be written as ${ }^{18)}$

$$
\ln \left(1-F I / F I_{\infty}\right)=A-L t
$$

where $F I_{\infty}$ is the fluorescence intensity of the liposomal solution after the addition of $10 \%$ Triton X-100 solution, and $L$ is the rate constant of $\mathrm{CF}$ leakage from liposomes. Here, $A=\left(1-F I_{0} / F I_{\infty}\right)$ and $F I_{0}$ is the initial fluorescence intensity. Figure 2 shows the typical plot of $\ln \left(1-F I / F I_{\infty}\right) v s$. $t$ for $\mathrm{CF}$ leakage from liposomes. In the case of DPPC-liposomes with or without FBS at near the phase transition temperature, a linear relationship was not established. Then, because a linear relation had been almost approved in the early stage of the leakage, the initial rate constant could be calculated for DPPC-liposomes. On the other hand, in the case of PEG-liposomes with or without FBS, the plot gave good straight lines according to Eq. 1.

Figure 3 shows the resulting $L$-values plotted as a function of temperature. The liposomes showed the maximum $L$-value at near the main phase transition temperature. The solute leakage from liposomes rapidly increases near the main phase transition temperature, and this phenomenon is responsible for the reduction of the stability of the boundary regions
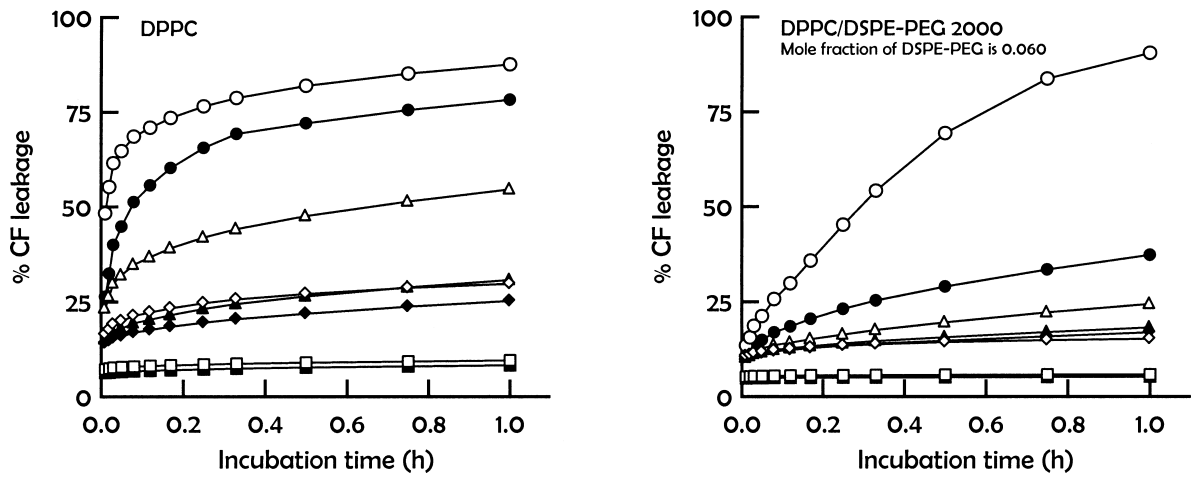

Fig. 1. Time-Courses of CF Leakage from Liposomes in the Absence (Closed Symbols) and Presence (Open Symbols) of FBS $\square: 30^{\circ} \mathrm{C}, \bullet \circ: 41^{\circ} \mathrm{C}, \boldsymbol{\Delta} \triangle: 44^{\circ} \mathrm{C}, \diamond: 50^{\circ} \mathrm{C}$.
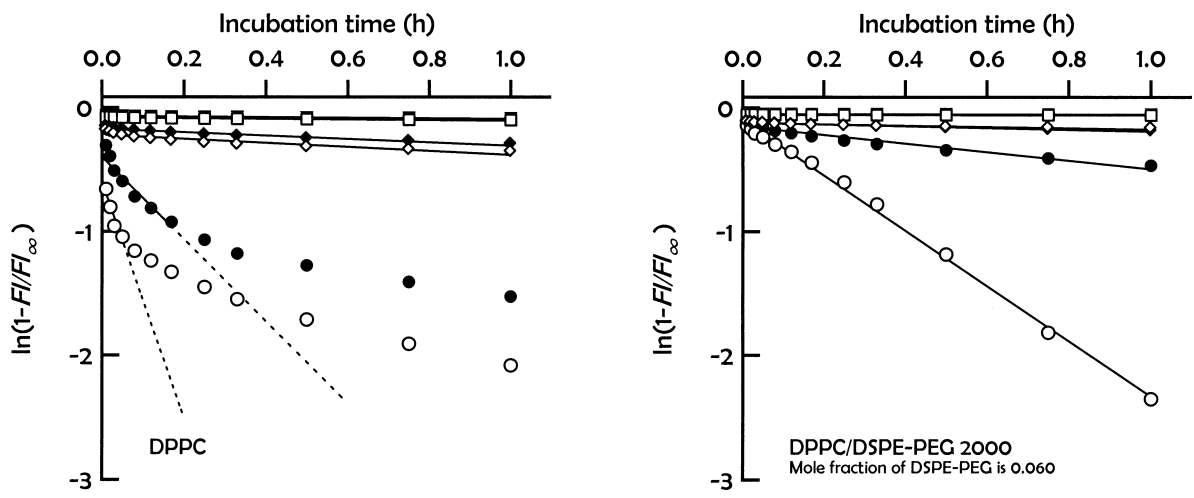

Fig. 2. Plots of $\ln \left(1-F I / F I_{\infty}\right)$ vs. $t$ According to Eq. 1 for CF Leakage from Liposomes

The symbols correspond to those in Fig. 1. 

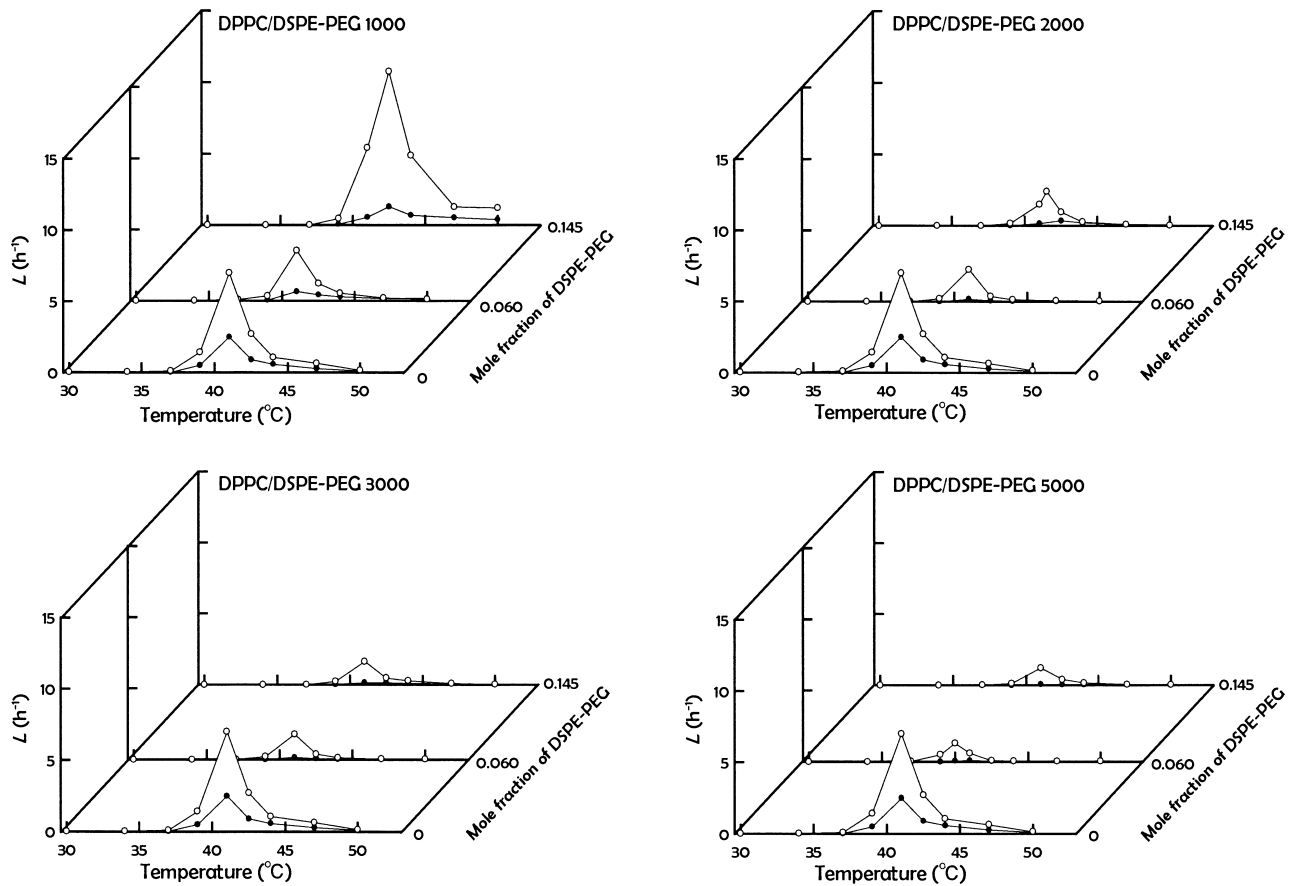

Fig. 3. Relationship between Rate Constant $(L)$ for CF Leakage from Liposomes and Temperature in the Absence (Closed Symbols) and Presence (Open Symbols) of FBS

in liposomal bilayer membranes owing to the formation of the discontinuity of the gel phase and liquid-crystalline phase. Furthermore, near the phase transition temperature, the $L$-values of the DPPC-liposomes with FBS were about 3 times larger than that of those without FBS, while $L$-values of the PEG-liposomes with FBS was 5 to 10 times larger than that of liposomes without FBS. The above results show that the phase separation at gel-liquid crystalline phase transition allowed proteins to interact with the liposomal bilayer membranes. The $\mathrm{CF}$ leakage from PEG-liposomes whose molecular weight in PEG units were above 2000 was suppressed compared with that of liposomes without DSPEPEG. In our previous papers, ${ }^{12,13)}$ we reported the effect of PEG-lipid on the membrane characteristics of liposomes. The addition of DSPE-PEG to DPPC-liposomes caused lateral phase separation in the gel and liquid-crystalline states, and the fluidity in the interfacial region of liposomal bilayer membranes was markedly increased. In addition, the permeability of liposomal bilayer membranes in PBS decreased compared with those without PEG. The PEG chains of DSPE-PEG are exposed from the liposomal surfaces when DSPE-PEG forms liposomal bilayer membranes with DPPC. ${ }^{7)}$ The exposed PEG chains shield the solute leakage from the liposomes. From these results, we concluded that the solute leakage from PEG-liposomes originates from the balance of the accelerating effect by the phase separation of the liposomal bilayer membranes and the shielding effect of PEG chains of the liposomal surface. Therefore, it is thought that the leakage of CF from PEG-liposomes whose molecular weight in PEG units was above 2000 was suppressed because the shielding effect due to the PEG chain was superior to the accelerating effect caused by the phase separation of liposomal bilayer membranes in FBS as well as the results in the PBS. Moreover, it was noteworthy that the leakage of $\mathrm{CF}$ from liposomes containing DSPE-PEG1000 with a $0.145 \mathrm{~mol}$ fraction was larger than that of liposomes without DSPEPEG. From this finding, it would be surmised that the phase separation in the bilayer membrane progressed due to the addition of a large amount of DSPE-PEG to DPPC-liposomes. The accelerating effect caused by the phase separation was superior to the shielding effect due to the PEG chain, which explains why the CF leakage increased in PEG-liposomes whose molecular weight in PEG units was 1000 with FBS.

Differential Scanning Calorimetry of Liposomal Bilayer Membranes Lipid bilayer membranes are in the lamellar gel state and the liquid crystalline state below and above the main phase transition temperature, respectively. The effects of FBS on the gel-liquid crystalline phase transition of liposomes were examined by differential scanning calorimetry (DSC) measurement. Figure 4 shows the DSC curves at $35-50{ }^{\circ} \mathrm{C}$ for PEG-liposomes in the absence and presence of FBS.

The DSC curves showed an endothermic peak at about $41{ }^{\circ} \mathrm{C}$ due to the phase transition temperature of DPPC. The gel-liquid crystalline phase transition temperature of liposomes containing DSPE-PEG with 0 and $0.060 \mathrm{~mol}$ fractions was irrelevant to the presence of FBS, while that of liposomes containing DSPE-PEG with $0.145 \mathrm{~mol}$ fractions was shifted to a higher temperature in the presence of FBS, except for DSPE-PEG5000. Here, it can explain the change in the phase transition temperature from the Gibbs free energy of the gel and the liquid-crystalline phases. ${ }^{19)}$ Two possible explanations have been given for the gel-liquid crystalline phase transition temperature of liposomes shifting to a higher temperature in the presence of FBS, (1) The Gibbs free energy of the gel state decreased by stabilizing the gel phase of liposomal bilayer membranes. (2) The Gibbs free energy of the liquid-crystalline state increased due to the destabilization of the liquid-crystalline phase of liposomal bilayer membranes. As for the rise of the phase transition temperature in 
PEG-liposomes, it is possible that these effects acted alone or both effects acted synergistic. Generally, the packing state of the lipid molecule in the bilayer membrane was significantly loosened by the change from the gel to liquid-crystalline states, and it is thought that the proteins invade the bilayer membranes in the liquid-crystalline state more easily than that in the gel state. Therefore, it can be judged that the liposomal bilayer membranes in the liquid-crystalline state becomes more unstable than that in the gel state due to the addition of FBS, and the gel-liquid crystalline phase transition temperature shifted to a higher temperature.

To evaluate the effects of FBS on the gel-liquid crystalline phase transition of liposomal bilayer membranes in further detail, the cooperative unit during the gel-liquid crystalline phase transition was calculated. ${ }^{20)}$ The ratio of the van't Hoff enthalpy change $\left(\Delta H_{\mathrm{vH}}\right)$ and calorimetric enthalpy change $(\Delta H)$ is known as the cooperative unit $\left(\Delta H_{\mathrm{vH}} / \Delta H\right)$ that represents the number of molecules going through the gel-liquid crystalline phase transition simultaneously. A reduction of the cooperative unit generally indicates that there is an increase in the heterogeneity among lipid molecules in the bilayer membranes during the phase transition. Table 1 summa-
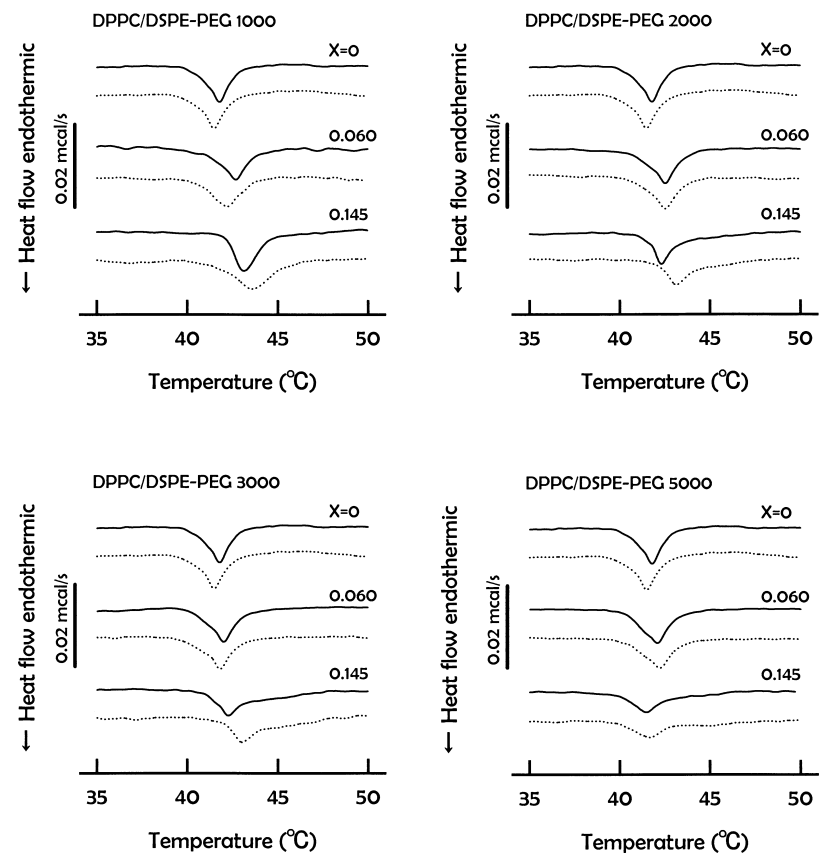

Fig. 4. DSC Thermograms for Liposomal Bilayer Membranes in the Absence (Solid Line) and Presence (Broken Line) of FBS rizes the transition enthalpies and cooperative units for liposomes in the absence and presence of FBS. The cooperative unit of liposomes with FBS had little change compared with that of liposomes without FBS. It follows from this that the addition of FBS hardly influences the interaction of DPPC molecules in the bilayer membranes. On the other hand, Tsunoda et al. ${ }^{21)}$ studied whether the effects of proteins, such as albumin and lysozyme, on the gel-liquid crystalline phase transition of negatively charged dipalmitoylphosphatidylglycerol (DPPG) liposomes was determined by DSC measurements, and the DSC peak area of liposomes decreased with the increasing adsorption of the proteins. This is because the proteins penetrate through DPPG-liposomes due to an electrostatic interaction and it weakened the interaction among DPPG molecules. These results indicate that neutral liposomes hardly change the heterogeneity among individual molecules during the gel-liquid crystalline phase transition.

\section{Conclusions}

We investigated the effects of FBS on the CF leakage from PEG-liposomes. The CF leakage from liposomes with FBS was much larger than that of liposomes without FBS at near the gel-liquid crystalline phase transition temperature. However, the cooperative units of lipid molecules during the gel-liquid crystalline phase transition of liposomes with FBS had little change compared with that of liposomes without FBS. From these results, it is suggested that the cooperative unit of liposomal bilayer membranes hardly change, so that FBS may attack the boundary region of the gel phase and liquid-crystalline phase by the hydrophobic interaction. Therefore, it was thought that the stability of the boundary region of the phase separation decreased for the attack of FBS, and the leakage of CF rapidly increased. On the other hand, FBS hardly influences the CF leakage from liposomes at a temperature away from the gel-liquid crystalline phase transition temperature. From above results, it was suggested to be able to make PEG-liposomes that had stability and temperaturesensitivity in the blood using the neutral lipid, such as DPPC. Knowledge of the experimental findings of the present study becomes a fundamental guide in the selection of lipids for the preparation of PEG-liposomes.

\footnotetext{
References

1) Bangham A. D., Standish M. M., Watkins J. C., J. Mol. Biol., 13, $238-252$ (1965)

2) Gregoriadis G., "Liposomes as Drug Carriers. Recent Trend and Progress," John Wiley \& Sons, New York, 1988.

3) Allen T. M., Chonn A., FEBS Lett., 223, $42-46$ (1987).
}

Table 1. Transition Enthalpy $(\Delta H)$ and Cooperative Unit $\left(\Delta H_{\mathrm{vH}} / \Delta H\right)$ for PEG-Liposomes

\begin{tabular}{|c|c|c|c|c|c|}
\hline & $\mathrm{X}_{\text {DSPE-PEG }}$ & $\begin{array}{c}\Delta H \text { in buffer } \\
(\mathrm{kcal} / \mathrm{mol})\end{array}$ & $\begin{array}{c}\Delta H \text { in FBS } \\
(\mathrm{kcal} / \mathrm{mol})\end{array}$ & $\begin{array}{l}\Delta H_{\mathrm{vH}} / \Delta H \\
\text { in buffer }\end{array}$ & $\begin{array}{c}\Delta H_{\mathrm{vH}} / \Delta H \\
\text { in FBS }\end{array}$ \\
\hline DPPC & & 6.8 & 6.4 & 51 & 55 \\
\hline \multirow[t]{2}{*}{ DPPC/DSPE-PEG 1000} & 0.060 & 6.4 & 6.7 & 44 & 46 \\
\hline & 0.145 & 5.3 & 4.9 & 80 & 76 \\
\hline \multirow[t]{2}{*}{ DPPC/DSPE-PEG 2000} & 0.060 & 5.3 & 5.4 & 85 & 77 \\
\hline & 0.145 & 5.3 & 5.5 & 47 & 42 \\
\hline \multirow[t]{2}{*}{ DPPC/DSPE-PEG 3000} & 0.060 & 5.5 & 5.5 & 75 & 70 \\
\hline & 0.145 & 5.6 & 5.9 & 42 & 40 \\
\hline \multirow[t]{2}{*}{ DPPC/DSPE-PEG 5000} & 0.060 & 6.2 & 5.9 & 62 & 57 \\
\hline & 0.145 & 4.8 & 4.4 & 45 & 39 \\
\hline
\end{tabular}


4) Allen T. M., Hansen C., Rutledge J., Biochim. Biophys. Acta, 981, 27-35 (1989).

5) Gabizon A., Papahadjopoulos D., Proc. Natl. Acad. Sci. U.S.A., 85, 6949-6953 (1988).

6) Klibanov A. L., Maruyama K., Torchilin V. P., Huang L., FEBS Lett., 268, 235-237 (1990).

7) Klibanov A. L., Maruyama K., Beckerleg A. M., Torchilin V. P., Huang L., Biochim. Biophys. Acta, 1062, 142-148 (1991).

8) Allen T. M., Hansen C., Martin F., Redemann C., Yau-Young A., Biochim. Biophys. Acta, 1066, 29-36 (1991).

9) Woodle M. C., Lasic D. D., Biochim. Biophys. Acta, 1113, 171-199 (1992).

10) Lasic D. D., Martin F. J., Gabizon A., Huang S. K., Papahadjopoulos D., Biochim. Biophys. Acta, 1070, 187-192 (1991).

11) Needham D., McIntosh T. J., Lasic D. D., Biochim. Biophys. Acta, 1108, $40-48$ (1992).

12) Hashizaki K., Itoh C., Sakai H., Yokoyama S., Taguchi H., Saito Y., Ogawa N., Abe M., Colloids Surf. B, 17, 275-282 (2000).
13) Hashizaki K., Taguchi H., Itoh C., Sakai H., Abe M., Saito Y., Ogawa N., Chem. Pharm. Bull., 51, 815-820 (2003).

14) Hashizaki K., Taguchi H., Itoh C., Sakai H., Abe M., Saito Y., Ogawa N., Chem. Pharm. Bull., 53, 27-31 (2005).

15) Yatvin M. B., Weinstein J. N., Dennis W. H., Blumenthal R., Science, 202, 1290-1293 (1978).

16) Senior J., Gregoriadis G., Life Sci., 30, 2123-2136 (1982).

17) Weinstein J. N., Klausner R. D., Innerarity T., Ralston E., Blumenthal R., Biochim. Biophys. Acta, 647, 270-284 (1981).

18) Komatsu H., Okada S., Biochim. Biophys. Acta, 1237, 169-175 (1995).

19) Murakami Y., "Chobunshikagaku no kiso to oyo," NTS Inc., Tokyo, 1996.

20) Kodama M., J. Jpn. Oil Chem. Soc., 39, 530-537 (1990).

21) Tsunoda T., Imura T., Kadota M., Yamazaki T., Yamauchi H., Kwon K. O., Yokoyama S., Sakai H., Abe M., Colloids Surf. B, 20, 155-163 (2001). 